\title{
Growth tightness for word hyperbolic groups
}

\author{
G. N. Arzhantseva ${ }^{1}$, I. G. Lysenok ${ }^{2}$ \\ 1 Section de Mathématiques, Université de Genève, CP 240, 1211 Genève 24, Switzerland \\ (e-mail: Goulnara.Arjantseva@math.unige.ch \\ 2 Steklov Institute of Mathematics, Gubkina str. 8, 117966 Moscow, Russia \\ (e-mail: lysionok@mi.ras.ru)
}

Received: 20 September 2001; in final form: 24 January 2002/

Published online: 5 September 2002 - (c) Springer-Verlag 2002

\begin{abstract}
We show that non-elementary word hyperbolic groups are growth tight. This means that, given such a group $G$ and a finite set $A$ of its generators, for any infinite normal subgroup $N$ of $G$, the exponential growth rate of $G / N$ with respect to the natural image of $A$ is strictly less than the exponential growth rate of $G$ with respect to $A$.
\end{abstract}

\section{Introduction}

Let $G$ be a finitely generated group and $A$ a finite set of generators for $G$. By $|x|$ we denote the geodesic length of an element $x \in G$ in the generators $A$, i.e. the length of a shortest word in the alphabet $A^{ \pm 1}$ representing $x$. Let $B(n)$ denote the ball $\{g \in G|| g \mid \leq n\}$ of radius $n$ in $G$.

The exponential growth rate of the pair $(G, A)$ is the limit

$$
\lambda(G, A)=\lim _{n \rightarrow \infty} \sqrt[n]{\# B(n)}
$$

where $\# X$ denotes the number of elements of a finite set $X$. The existence of the limit follows from the submultiplicativity property of the function $\# B(n): \# B(m+n) \leq \# B(n) \# B(m)$ for any $n, m \geq 0$, see for example [8, VI.C]. The uniform exponential growth rate $\lambda(G)$ of $G$ is the infinum $\inf _{A} \lambda(G, A)$ over all finite generating sets $A$ of $G$.

We say that a pair $(G, A)$ is growth tight if $\lambda(G, A)>\lambda(G / N, \underline{A})$ for all infinite normal subgroups $N$ of $G$, with $\underline{A}$ denoting the canonical image of $A$ in $G / N$. (This is a modification of the definition in [5].) Observe that, for

The work has been supported by the Swiss National Science Foundation. The second author has been supported also by INTAS, grant No. 97-1259 
a torsion-free group $G$, "infinite" means "any nontrivial" in this definition and it coincides then with one given in [5]. On the other hand, if $N$ is finite it is not hard to see that we always have $\lambda(G, A)=\lambda(G / N, \underline{A})$.

It is known that for a free group $F_{k}$ of rank $k \geq 2$ and for a free basis $X_{k}$ of $F_{k}$, the pair $\left(F_{k}, X_{k}\right)$ is growth tight. The direct product $F_{k} \times F_{k}$ generated by $X=\left(X_{k} \times\{1\}\right) \cup\left(\{1\} \times X_{k}\right)$ is an example of a pair which is not growth tight [5].

For results and applications related to the exponential growth rate and the growth tightness property, see $[5,8,9]$. We mention here that the exponential growth rate of the pair $(G, A)$ and its logarithm, sometimes called the entropy of the pair $(G, A)$, give rise to bounds of the growth of the volume of balls in a Riemannian manifold $M$ with fundamental group $G$ as well as to bounds of the topological entropy of the geodesic flow on $M$, see [6, Sect. 5.B].

Recall that a group $G$ is called Hopfian if every epimorphism $G \rightarrow G$ is an isomorphism, i.e. there is no a proper quotient $G / N$ of $G$ isomorphic to $G$. As an immediate consequence of the above definitions we have the following

Observation. Let $G$ be a finitely generated group. If there exists a finite generating set $A$ of $G$ such that $\lambda(G, A)=\lambda(G)$ and if $(G, A)$ is growth tight, then $G / N$ cannot be isomorphic to $G$ for any infinite normal subgroup $N$ of $G$.

In this paper, we restrict our attention to the class of word hyperbolic groups $G$ in the sense of M.Gromov [7]. Note that any word hyperbolic group is finitely generated and even finitely presented [7, 2.1A, 2.2A], [4, Proposition 4.17]. It is known also that there are only finitely many conjugacy classes of finite subgroups in a word hyperbolic group $G$, see for example [2, Ch.III. $\Gamma$, Theorem 3.2]. This implies in particular that $G / N$ is not isomorphic to $G$ for any nontrivial finite normal subgroup $N$ of $G$. Indeed, if such an isomorphism $\phi: G \rightarrow G / N$ would exist then we could define an infinite strictly increasing sequence $N_{i}(i \geq 0)$ of finite subgroups of $G$ by $N_{0}=N$ and $N_{i+1}=\psi^{-1} \phi\left(N_{i}\right)$ where $\psi: G \rightarrow G / N$ is the canonical epimorphism.

Thus, the observation provides a possible way to prove the Hopf property for word hyperbolic groups. Note that torsion-free word hyperbolic groups are already known to be Hopfian [10]. For word hyperbolic groups with torsion, the question whether or not they are Hopfian is still open.

Our main result concerns the second condition in the observation above and gives an affirmative answer to the question about growth tightness of word hyperbolic groups, posed by R. Grigorchuk and P. de la Harpe [5]. Recall that a word hyperbolic group is called elementary if it is either finite or a finite extension of the infinite cyclic group. 
Theorem 1. Let $G$ be a non-elementary word hyperbolic group and A any finite set of generators for $G$. Then, for any infinite normal subgroup $N$ of $G$,

$$
\lambda(G, A)>\lambda(G / N, \underline{A}),
$$

where $\underline{A}$ is the canonical image of $A$ in $G / N$.

To prove the theorem we first observe that the growth tightness of $G$ is related to the growth tightness of certain languages over $A^{ \pm 1}$ and we obtain a stronger result (Theorem 2 below) which implies Theorem 1 .

We fix an arbitrary linear ordering on the alphabet $A^{ \pm 1}$. A word $w$ in the alphabet $A^{ \pm 1}$ is called geodesic if $w$ has the minimal possible length among all the words representing the same element of $G$. If $w$ is geodesic and is minimal in the induced lexical ordering, among all geodesic words representing the same element of $G$, it is called shortlex geodesic. By $\mathcal{L}$ we denote the set of all shortlex geodesic words in $A^{ \pm 1}$. Clearly, every element $x \in G$ has a unique representative in $\mathcal{L}$ which we denote $\bar{x}$. It is obvious also that any subword of a shortlex geodesic word is itself shortlex geodesic.

Definition 1. Let $C>0$. We say that two elements $x, y \in G$ are $C$-close if there are $u, v \in G$ such that $x=u y v$ and $|u|,|v| \leq C$. We say that $x$ $C$-contains $y$ if $\bar{x}$ has a subword representing an element which is $C$-close to $y$. For a given $w \in G$, we denote

$$
Z_{w, C}=\{x \in G \mid x C \text {-contains } w\} .
$$

We define the exponential growth rate of the set $G \backslash Z_{w, C}$ by

$$
\lambda_{w, C}=\limsup _{n \rightarrow \infty} \sqrt[n]{\#\left(B(n) \backslash Z_{w, C}\right)}
$$

Theorem 2. Let $G$ be a non-elementary word hyperbolic group and $A$ a finite set of generators for $G$. Let $\lambda=\lambda(G, A)$ be the exponential growth rate of $G$ with respect to $A$. Then there is a number $C=C(G, A)$ such that $\lambda_{w, C}<\lambda$ for any $w \in G$.

Note that Theorem 2 is of somewhat technical nature because the definition of $\lambda_{w, C}$ uses the language of shortlex geodesic words in $G$. But it easily implies a stronger result which does not make use of any specific language. To formulate this result, we use, in an informal sense, "the strongest" form of the relation " $x C$-contains $y$ " which leads to the "smallest possible" appropriate set $Z_{w, C}$ and, consequently, to the "largest possible" appropriate parameter $\lambda_{w, C}$. 
We say that $x$ strongly $C$-contains $y$ if any geodesic word representing $x$ contains a subword which is $C$-close to $y$. Let

$$
\bar{Z}_{w, C}=\{x \in G \mid x \text { strongly } C \text {-contains } w\}
$$

and let $\bar{\lambda}_{w, C}$ be the exponential growth rate of the set $G \backslash \bar{Z}_{w, C}$, defined as in (1).

Theorem 3. Let $G$ be a non-elementary word hyperbolic group and $A$ a finite set of generators for $G$. Let $\lambda=\lambda(G, A)$ be the exponential growth rate of $G$ with respect to $A$. Then there is a number $C=C(G, A)$ such that $\bar{\lambda}_{w, C}<\lambda$ for any $w \in G$.

Acknowledgments. This work has been done during the second author's visit to the University of Geneva in May 2001. He thanks P. de la Harpe for hospitality. We thank also the referee for useful comments.

\section{Preliminaries}

\subsection{Languages}

Let $L$ be a language over a finite alphabet $X$, i.e. $L$ is any set of words in the alphabet $X$. We denote

$$
B_{L}(n)=\{x \in L|| x \mid \leq n\}, \quad \lambda_{L}=\limsup _{n \rightarrow \infty} \sqrt[n]{\# B_{L}(n)}
$$

and, for any word $w \in L$, we define

$$
L_{w}=\{x \in L \mid x \text { contains } w \text { as a subword }\} .
$$

We say that $L$ is growth tight if $\lambda_{L \backslash L_{w}}<\lambda_{L}$ for any $w \in L$.

Now let $G$ be a finitely generated group, $A$ a finite set of its generators, and $\mathcal{L}$ a language of shortlex geodesic words in the alphabet $A^{ \pm 1}$. Assume that $\mathcal{L}$ is growth tight. It is then easy to see that the pair $(G, A)$ is growth tight. Indeed, let $N$ be a nontrivial normal subgroup of $G$. Choosing a word $w \in \mathcal{L}$ representing a nontrivial element of $N$ we see that $\mathcal{L}_{G / N} \subseteq \mathcal{L} \backslash \mathcal{L}_{w}$ where $\mathcal{L}_{G / N}$ is the language of shortlex geodesic words in $G / N$. This obviously implies

$$
\lambda(G / N, \underline{A})=\lambda_{\mathcal{L}_{G / N}} \leq \lambda_{\mathcal{L} \backslash \mathcal{L}_{w}}<\lambda_{\mathcal{L}}=\lambda(G, A) .
$$

(Here $\underline{A}$, as above, denotes the canonical image of $A$ in $G / N$.)

Thus to prove growth tightness of $G$ it suffices to prove growth tightness of the language $\mathcal{L}$. This approach works well for example for proving growth tightness of finitely generated free groups. But in general, we observe that 
growth tightness of $\mathcal{L}$ implies that $\lambda(G / N, \underline{A})<\lambda(G, A)$ for any nontrivial normal subgroup $N$ of $G$; and as we have mentioned above, for finite $N$ we have the equality. This means in particular that the language $\mathcal{L}$ is not growth tight if $G$ has a nontrivial finite normal subgroup.

We use a slight modification of this approach for word hyperbolic groups. Namely, for $C>0$ we define

$$
\begin{gathered}
L_{w, C}=\{x \in L \mid x \text { has a subword representing an element of } G \\
\text { which is } C \text {-close to } w\} .
\end{gathered}
$$

We say that $L$ is growth quasitight if there exists $C=C(G, A)>0$ such that for any $w \in L$ we have $\lambda_{L}>\lambda_{L \backslash L_{w, C}}$.

Now observe that growth quasitightness of $\mathcal{L}$ implies growth tightness of $G$. Let $N$ be an infinite normal subgroup of $G$. We take a word $w \in \mathcal{L}$ representing an element of $N$ and of length greater than $4 C$. Suppose that a word $x \in \mathcal{L}$ has a subword $y$ representing an element which is $C$-close to $w$, that is, $y=u w v$ in $G$ for some $u, v$ with $|u|,|v| \leq C$. We have $|y| \geq|w|-|u|-|v|>2 C$. This implies that $x$ is not geodesic in $G / N$ as its subword $y$ is equal in $G / N$ to a shorter word $u v$. Hence $\mathcal{L}_{G / N} \subseteq \mathcal{L} \backslash \mathcal{L}_{w, C}$ and again we have $\lambda(G / N, \underline{A})=\lambda_{\mathcal{L}_{G / N}} \leq \lambda_{\mathcal{L} \backslash \mathcal{L}_{w, C}}<\lambda_{\mathcal{L}}=\lambda(G, A)$.

As a consequence, we obtain that Theorem 2 implies Theorem 1.

\subsection{Auxiliary results on word hyperbolic groups}

We fix a group $G$ and a finite set $A$ of generators for $G$ for the rest of the paper. All words are assumed to be in the alphabet $A^{ \pm 1}$. We shall make no essential distinction between words and elements of $G$. If $w$ is a word then the notation $w \equiv x y$ means that $w$ can be decomposed, as a word, into a product of two words which represent elements $x, y \in G$.

There are known several properties of a group $G$ which are equivalent to its word hyperbolicity. For practical purposes, one of the most useful is the property of geodesic triangles in the Cayley graph of $G$ being $\delta$-thin, see [7, 6.3], [4, Proposition 2.21]. In the combinatorial language, it can be formulated as follows. Recall that the Gromov inner product of two elements $x, y \in G$ with respect to the identity element of $G$ is defined to be

$$
(x \mid y)=\frac{1}{2}\left(|x|+|y|-\left|x^{-1} y\right|\right) .
$$

Then $G$ is word hyperbolic if and only if there is a number $\delta \geq 0$ such that the following is true:

(H1) for any two geodesic words $u$ and $v$, if $u \equiv u_{1} u_{2}, v \equiv v_{1} v_{2}$ and $\left|u_{1}\right|=\left|v_{1}\right| \leq(u \mid v)$ then $\left|u_{1}^{-1} v_{1}\right| \leq \delta$. 
From now on, we assume $G$ to be word hyperbolic and fix a number $\delta \geq 0$ such that (H1) holds.

As a consequence of (H1), we have another property of $G$ which is in fact original Gromov's definition, see [7, 6.3B], [4, 2.21]:

$$
(x \mid y) \geq \min \{(x \mid z),(y \mid z)\}-2 \delta \text { for any } x, y, z \in G .
$$

Lemma 1. For any $x, y, z \in G$ the following assertions hold.

(a) If $\left(x^{-1} \mid y\right) \leq r,\left(y^{-1} \mid z\right) \leq t$ and $|y|>r+t+2 \delta$ then $\left((x y)^{-1} \mid z\right) \leq$ $t+2 \delta$ and $\left(x^{-1} \mid y z\right) \leq r+2 \delta$. We have also

$$
|x y z| \geq|x|+|y|+|z|-2(r+t+2 \delta) .
$$

(b) If $x$ is a shortest representative in its conjugacy class and $|x| \geq 2 \delta+2$ then $\left(x^{-1} \mid x\right)<\delta+1$.

Proof. (a): Let $\left(x^{-1} \mid y\right) \leq r,\left(y^{-1} \mid z\right) \leq t$ and $|y|>r+t+2 \delta$. We have

$$
\left(y^{-1} \mid(x y)^{-1}\right)=\frac{1}{2}(|y|+|x y|-|x|)=|y|-\left(x^{-1} \mid y\right)>t+2 \delta .
$$

Assuming $\left((x y)^{-1} \mid z\right)>t+2 \delta$, by (H2) we get $\left(y^{-1} \mid z\right)>t$ contrary to the hypothesis. Hence $\left((x y)^{-1} \mid z\right) \leq t+2 \delta$. Similarly, observing that $(y \mid y z)=|y|-\left(y^{-1} \mid z\right)>r+2 \delta$ from $\left(x^{-1} \mid y\right) \leq r$ we deduce by (H2) that $\left(x^{-1} \mid y z\right) \leq r+2 \delta$.

Now using $\left((x y)^{-1} \mid z\right) \leq t+2 \delta$ and $\left(x^{-1} \mid y\right) \leq r$, we obtain

$$
|x y z| \geq|x y|+|z|-2(t+2 \delta) \geq|x|+|y|+|z|-2(r+t+2 \delta) .
$$

(b): Let $x$ be a shortest representative in its conjugacy class and $|x| \geq$ $2 \delta+2$. Assume that $\left(x^{-1} \mid x\right) \geq \delta+1$. Let $\tilde{x}$ be a geodesic word representing $x$ and let $\tilde{x} \equiv x_{1} x_{2} x_{3}$ where $\left|x_{1}\right|=\left|x_{3}\right|=\delta+1$. By (H1), $\left|x_{3} x_{1}\right| \leq \delta$. Then $\left|x_{3} x x_{3}^{-1}\right|=\left|x_{3} x_{1} x_{2}\right| \leq\left|x_{2}\right|+\delta<|x|$ contrary to the choice of $x$.

Definition 2 (a refinement of Definition 1). Let $x, y \in G$ and $0 \leq t_{1} \leq$ $t_{2} \leq|x|$. We say that $x\left(r, t_{1}, t_{2}\right)$-contains $y$ if $\bar{x} \equiv x^{\prime} z x^{\prime \prime}$ where $z$ is $r$-close to $y$ and $\left|x^{\prime}\right| \geq t_{1}$ and $\left|x^{\prime} z\right| \leq t_{2}$. (This is equivalent to the condition that if we divide $\bar{x}$ into segments $\bar{x} \equiv x_{1} w x_{2}$ with $\left|x_{1}\right|=t_{1},\left|x_{1} w\right|=t_{2}$ then $w$ $r$-contains $y$.)

\section{Lemma 2.}

(a) If $x$ is $r$-close to $y$ and $y$ is t-close to $z$ then $x$ is $(r+t)$-close to $z$.

(b) Let $\left|x^{-1} y\right| \leq r$ and $\tilde{x}, \tilde{y}$ be geodesic words representing $x$ and $y$, respectively. If $\tilde{x} \equiv x_{1} x_{2}$ then $\tilde{y} \equiv y_{1} y_{2}$ for some $y_{1}$, $y_{2}$ where $\left|x_{1}^{-1} y_{1}\right| \leq$ $r+\delta$ and $x_{2}$ is $(r+\delta)$-close to $y_{2}$. 
(c) Let $x$ and $y$ be $r$-close and let $\tilde{x}$ and $\tilde{y}$ be any geodesic words representing $x$ and $y$, respectively. Then the following assertions are true. If $\tilde{x} \equiv x_{1} x_{2}$ then $\tilde{y} \equiv y_{1} y_{2}$ for some $y_{1}$ and $y_{2}$ where $x_{i}$ is $(r+2 \delta)$-close to $y_{i}$ $(i=1,2)$. Any subword of $\tilde{x}$ is $(r+2 \delta)$-close to some subword of $\tilde{y}$ and, in particular, is $(r+2 \delta)$-contained in $y$.

(d) If $\left(x^{-1} \mid y\right) \leq r$ then both $x$ and $y$ are $(r+\delta+1)$-contained in $x y$. Moreover, if $w$ is a geodesic word representing $x y$ then $w \equiv x_{1} y_{1}$ where $\left|x^{-1} x_{1}\right| \leq r+\delta+1$ and $\left|y y_{1}^{-1}\right| \leq r+\delta+1$.

(e) If $x$ is $\left(r, t_{1}, t_{2}\right)$-contained in $y$, $t_{1}^{\prime} \leq t_{2}^{\prime} \leq|y|$ and $\left|t_{i}-t_{i}^{\prime}\right| \leq s(i=1,2)$ then $x$ is $\left(r+s, t_{1}^{\prime}, t_{2}^{\prime}\right)$-contained in $y$.

(f) If $x$ is $\left(r, t_{1}, t_{2}\right)$-contained in $y$, and $y$ is $s$-close to $z$ then $x$ is $(r+s+$ $\left.2 \delta, t_{1}^{\prime}, t_{2}^{\prime}\right)$-contained in $z$ for some $t_{1}^{\prime}$, $t_{2}^{\prime}$ with $\left|t_{i}-t_{i}^{\prime}\right| \leq 2 s+2 \delta$. In particular, by (e), if $t_{2} \leq|z|$ then $x$ is $\left(r+3 s+4 \delta, t_{1}, t_{2}\right)$-contained in $z$.

Proof. Assertion (a) easily follows from Definition 1.

(b): Let $\tilde{x} \equiv x_{1} x_{2}$ and $\tilde{y} \equiv y_{1} y_{2}$. Notice that $x_{2}=x_{1}^{-1} y_{1} \cdot y_{2} \cdot\left(x^{-1} y\right)^{-1}$. Hence if $\left|x_{1}^{-1} y_{1}\right| \leq r+\delta$ then by Definition $1, x_{2}$ and $y_{2}$ are $(r+\delta)$-close. So it suffices to find a factorization $\tilde{y} \equiv y_{1} y_{2}$ with $\left|x_{1}^{-1} y_{1}\right| \leq r+\delta$.

If $\left|x_{1}\right| \leq(x \mid y)$ then by (H1), for $\tilde{y} \equiv y_{1} y_{2}$ with $\left|y_{1}\right|=\left|x_{1}\right|$ we have $\left|x_{1}^{-1} y_{1}\right| \leq \delta$. Let $\left|x_{1}\right|>(x \mid y)$. Since $(x \mid y)+\left(x^{-1} \mid x^{-1} y\right)=|x|$ we have $\left|x_{2}\right|<\left(x^{-1} \mid x^{-1} y\right)$. Let $w$ be a geodesic word representing $x^{-1} y$ and let $w \equiv w_{1} w_{2}$ where $\left|w_{1}\right|=\left|x_{2}\right|$. Then by (H1), $\left|x_{2} w_{1}\right| \leq \delta$ and hence $\left|x_{1}^{-1} y\right|=\left|x_{2} x^{-1} y\right| \leq\left|x_{2} w_{1}\right|+\left|w_{2}\right| \leq \delta+r$. In this case, we can take $y_{1}=y$ and $y_{2}=1$.

(c): Let $x=u y v$ where $|u|,|v| \leq r$. Denoting $z=x v^{-1}$ we have $\left|x^{-1} z\right|=|v| \leq r$ and $\left|z y^{-1}\right|=|u| \leq r$.

We prove the first assertion. Let $\tilde{x} \equiv x_{1} x_{2}$. Using the arguments from the proof of (b) we find $z_{1}$ and $z_{2}$ such that $\tilde{z} \equiv z_{1} z_{2}$ and either $\left|x_{1}^{-1} z_{1}\right| \leq \delta$ or $z_{1}=z$ and $\left|x_{1}^{-1} z_{1}\right| \leq r+\delta$. If $z_{1}=z$ then $x_{1}=u y\left(x_{1}^{-1} z_{1}\right)^{-1}$ and we can take $y_{1}=y$. Otherwise, the application of (b) with $x:=z^{-1}, y:=y^{-1}$ gives a factorization $\tilde{y} \equiv y_{1} y_{2}$ with $\left|z_{2} y_{2}^{-1}\right| \leq r+\delta$. Then $x_{1}=u y_{1} w$ where

$w=y_{1}^{-1} u^{-1} x_{1}=y_{1}^{-1} u^{-1} z \cdot z^{-1} \cdot x_{1}=y_{2} \cdot z_{2}^{-1} z_{1}^{-1} \cdot x_{1}=\left(z_{2} y_{2}^{-1}\right)^{-1}\left(x_{1}^{-1} z_{1}\right)^{-1}$

and hence $|w| \leq r+2 \delta$.

To prove the second assertion, we observe that the factorization $\tilde{y} \equiv y_{1} y_{2}$ given in the proof of (b), and hence in the previous argument, is monotone with respect to the factorization $\tilde{x} \equiv x_{1} x_{2}$; i.e., if $\tilde{x} \equiv x_{1}^{\prime} x_{2}^{\prime}$ and $\left|x_{1}^{\prime}\right| \geq$ $\left|x_{1}\right|$ then, for the corresponding $y_{1}^{\prime}$, we have $\left|y_{1}^{\prime}\right| \geq\left|y_{1}\right|$. This and the previous argument imply that if $\tilde{x} \equiv x_{1} x_{2} x_{3}$ then there are $y_{1}, y_{2}, y_{3}$ such that $\tilde{y} \equiv y_{1} y_{2} y_{3}$ and $x_{1}=u y_{1} w, x_{1} x_{2}=u y_{1} y_{2} w^{\prime}$ for some $w, w^{\prime}$ with $|w|,\left|w^{\prime}\right| \leq r+2 \delta$. But then $x_{2}=w^{-1} y_{2} w^{\prime}$ thus finishing the proof of (c). 
(d): If $\left|u^{-1} v\right| \leq t$ or $\left|u v^{-1}\right| \leq t$ then, obviously, $u$ is $t$-close to $v$. So the first assertion follows from the second.

Suppose that $\left(x^{-1} \mid y\right) \leq r$ and $w$ is a geodesic representative for $x y$. We choose a geodesic representative $\tilde{x}$ for $x$ and let $w \equiv x_{1} y_{1}, \tilde{x} \equiv x_{2} x_{3}$ where $(x \mid w)-1<\left|x_{1}\right|=\left|x_{2}\right| \leq(x \mid w)$. By (H1), $\left|x_{2}^{-1} x_{1}\right| \leq \delta$. Since $(x \mid w)+\left(x^{-1} \mid y\right)=|x|$ we have $\left|x_{3}\right|<\left(x^{-1} \mid y\right)+1$ and hence

$$
\left|x^{-1} x_{1}\right|=\left|x_{3}^{-1} \cdot x_{2}^{-1} x_{1}\right|<r+\delta+1 .
$$

It remains to notice that $y y_{1}^{-1}=x^{-1} x_{1}$.

(e): easily follows from Definition 2.

(f): Let $x$ be $\left(r, t_{1}, t_{2}\right)$-contained in $y$, and $y$ be $s$-close to $z$. By Definition $2, \bar{y} \equiv y_{1} x_{1} y_{2}$ where $x_{1}$ is $r$-close to $x,\left|y_{1}\right| \geq t_{1}$ and $\left|y_{1} x_{1}\right| \leq t_{2}$. Let $y=u z v$ where $|u|,|v| \leq s$. The proof of (c) shows that $\bar{z}$ may be decomposed as $\bar{z} \equiv z_{1} x_{2} z_{2}$ where $y_{1}=u z_{1} w, y_{1} x_{1}=u z_{1} x_{2} w^{\prime}$ and $x_{1}=w^{-1} x_{2} w^{\prime}$ for some $w$ and $w^{\prime}$ with $|w|,\left|w^{\prime}\right| \leq s+2 \delta$. By (a), $x_{2}$ is $(r+s+2 \delta)$-close to $x$. We have ||$y_{1}|-| z_{1}|| \leq 2 s+2 \delta$ and ||$y_{1} x_{1}|-| z_{1} x_{2}|| \leq 2 s+2 \delta$. Hence $x$ is $\left(r+s+2 \delta, t_{1}^{\prime}, t_{2}^{\prime}\right)$-contained in $z$ where $t_{1}^{\prime}=\max \left\{0, t_{1}-2 s-2 \delta\right\}$ and $t_{2}^{\prime}=\min \left\{|z|, t_{2}+2 s+2 \delta\right\}$.

Lemma 3. There is a number $D=D(G, A)$ with the following property. Given any $u, w \in G$ there exists a word $z \in \mathcal{L}$ such that $z \equiv z_{1} z_{2}$ for some $z_{1}, z_{2}$ with $\left|u^{-1} z_{1}\right| \leq D$ and $\left|w z_{2}^{-1}\right| \leq D$.

Proof. By [7, 5.1B], there are infinitely many conjugacy classes of elements in a non-elementary word hyperbolic group. We choose any $v_{0} \in G$ such that $\left|v_{0}\right| \geq 14 \delta+2$ and $v_{0}$ is a shortest representative in its conjugacy class. Let $H=\left\langle v_{0}\right\rangle$. Observe that $H$ has infinite index in $G$ since $G$ is nonelementary. Then there are infinitely many double cosets $H x H$ in $G$, as $H$ is a quasiconvex subgroup of infinite index in a word hyperbolic group, see for example [1, 2.3 and Proposition 1]. We choose $x \in G$ such that $|x| \geq\left|v_{0}\right|+2 \delta+1$ and $x$ is a shortest representative in the double coset $H x H$.

By the choice of $x$, we have $\left|v_{0}^{ \pm 1} x^{ \pm 1}\right| \geq|x|$. This implies that $\left(v_{0}^{ \pm 1} \mid x^{ \pm 1}\right)$ $\leq \frac{1}{2}\left|v_{0}\right|$. Since $|x|>\left|v_{0}\right|+2 \delta$, by Lemma 1(a) we get $\left(v_{0}^{ \pm 1} \mid x^{ \pm 1} v_{0}^{ \pm 1}\right) \leq$ $\frac{1}{2}\left|v_{0}\right|+2 \delta$ and

$$
\left|v_{0}^{ \pm 1} x v_{0}^{ \pm 1}\right| \geq|x|-4 \delta \geq 12 \delta+2 .
$$

Hence for $\varepsilon= \pm 1$ we obtain

$$
\left(v_{0}^{\varepsilon} \mid v_{0}^{\varepsilon} x^{ \pm 1} v_{0}^{ \pm 1}\right)=\left|v_{0}\right|-\left(v_{0}^{-\varepsilon} \mid x^{ \pm 1} v_{0}^{ \pm 1}\right) \geq \frac{1}{2}\left|v_{0}\right|-2 \delta .
$$

Now by Lemma 1 (b) and (H2), we have either $\left(u^{-1} \mid v_{0}\right)<3 \delta+1$ or $\left(u^{-1} \mid v_{0}^{-1}\right)<3 \delta+1$ and either $\left(w \mid v_{0}\right)<3 \delta+1$ or $\left(w \mid v_{0}^{-1}\right)<3 \delta+1$. Let $\left(u^{-1} \mid v_{0}^{\varepsilon}\right)<3 \delta+1$ and $\left(w \mid v_{0}^{\nu}\right)<3 \delta+1$. 
If $\left(u^{-1} \mid v_{0}^{\varepsilon} x v_{0}^{\nu}\right) \geq 5 \delta+1$, by (H2) with $x:=v_{0}^{\varepsilon}, y:=u^{-1}$ and $z:=$ $v_{0}^{\varepsilon} x v_{0}^{\nu}$ we would have

$$
\left(u^{-1} \mid v_{0}^{\varepsilon}\right) \geq \min \left\{\frac{1}{2}\left|v_{0}\right|-2 \delta, 5 \delta+1\right\}-2 \delta \geq 3 \delta+1,
$$

obtaining a contradiction. Hence

$$
\left(u^{-1} \mid v_{0}^{\varepsilon} x v_{0}^{\nu}\right)<5 \delta+1 .
$$

Similarly, we see that $\left(\left(v_{0}^{\varepsilon} x v_{0}^{\nu}\right)^{-1} \mid w\right)<5 \delta+1$. Since $\left|v_{0}^{\varepsilon} x v_{0}^{\nu}\right| \geq 12 \delta+2$, by Lemma 1(a),

$$
\left(\left(u v_{0}^{\varepsilon} x v_{0}^{\nu}\right)^{-1} \mid w\right)<7 \delta+1 .
$$

By Lemma 2(d), $\overline{u v_{0}^{\varepsilon} x v_{0}^{\nu}} \equiv u_{1} y$ where $\left|u^{-1} u_{1}\right| \leq 6 \delta+2$ and $\left|v_{0}^{\varepsilon} x v_{0}^{\nu} y^{-1}\right|$ $\leq 6 \delta+2$. The last inequality implies

$$
|y| \leq\left|v_{0}^{\varepsilon} x v_{0}^{\nu}\right|+6 \delta+2 \leq|x|+2\left|v_{0}\right|+6 \delta+2 .
$$

Again by Lemma 2(d), $\overline{u v_{0}^{\varepsilon} x v_{0}^{\nu} w} \equiv u_{2} w_{1}$ where $\left|\left(u v_{0}^{\varepsilon} x v_{0}^{\nu}\right)^{-1} u_{2}\right| \leq 8 \delta+2$ and $\left|w w_{1}^{-1}\right| \leq 8 \delta+2$. By Lemma 2(b), $u_{2} \equiv u_{3} y_{1}$ where $\left|u_{1}^{-1} u_{3}\right| \leq 9 \delta+2$ and $y_{1}$ is $(9 \delta+2)$-close to $y$. We have $\overline{u v_{0}^{\varepsilon} x v_{0}^{\nu} w} \equiv u_{3} y_{1} w_{1}$ where

$$
\left|u^{-1} u_{3}\right| \leq\left|u^{-1} u_{1}\right|+\left|u_{1}^{-1} u_{3}\right| \leq 15 \delta+4
$$

and

$\left|w\left(y_{1} w_{1}\right)^{-1}\right| \leq\left|w w_{1}^{-1}\right|+\left|y_{1}\right| \leq 8 \delta+2+|y|+2(9 \delta+2) \leq|x|+2\left|v_{0}\right|+32 \delta+8$.

Therefore, we can take $z=\overline{u v_{0}^{\varepsilon} x v_{0}^{\nu} w}, z_{1}=u_{3}, z_{2}=y_{1} w_{1}$ and $D=$ $|x|+2\left|v_{0}\right|+32 \delta+8$.

Lemma 4. There are numbers $E=E(G, A)$ and $\kappa=\kappa(G, A)>0$ with the following property. Let $w \in G$ be any element. Let

$$
X_{w}=\left\{x \in G|| w^{-1} x_{1} \mid \leq E \text { for some initial segment } x_{1} \text { of } \bar{x}\right\} .
$$

Then, for any $n \geq|w|$,

$$
\#\left(X_{w} \cap B(n)\right) \geq \kappa \# B(n-|w|) .
$$

Proof. We take $E=D$ and $\kappa=\frac{1}{(\# B(2 D))^{2}}$ where $D$ is as in Lemma 3. If $|w| \leq n<|w|+2 D$ then $\kappa \# B(n-|w|)<1$ and $\#\left(X_{w} \cap B(n)\right) \geq 1$ as $w \in X_{w}$; so the required inequality holds. Let $n \geq|w|+2 D$. For a given $w$ and any $u \in B(n-|w|-2 D)$ we take $z_{u} \equiv z_{1} z_{2} \in \mathcal{L}$ by Lemma 3 where $\left|w^{-1} z_{1}\right| \leq D$ and $\left|u z_{2}^{-1}\right| \leq D$. Clearly we have $z_{u}=w y_{u} u$ for some $y_{u} \in G$ with $\left|y_{u}\right| \leq 2 D$. In particular, $z_{u} \in X_{w} \cap B(n)$. 
By the pigeon hole principle, for some subset $U \subseteq B(n-|w|-2 D)$ with $\# U \geq \frac{1}{\#\left\{y_{u}\right\}} \# B(n-|w|-2 D)$ we have $y_{u_{1}}=y_{u_{2}}$ for all $u_{1}, u_{2} \in U$. It follows that $z_{u_{1}} \neq z_{u_{2}}$ for $u_{1}, u_{2} \in U, u_{1} \neq u_{2}$. Hence

$\#\left(X_{w} \cap B(n)\right) \geq \frac{1}{\#\left\{y_{u}\right\}} \# B(n-|w|-2 D) \geq \frac{1}{\# B(2 D)} \# B(n-|w|-2 D)$.

By submultiplicativity of $\# B(n)$,

$$
\# B(n-|w|-2 D) \geq \frac{1}{\# B(2 D)} \# B(n-|w|)
$$

and we finally have

$$
\#\left(X_{w} \cap B(n)\right) \geq \frac{1}{(\# B(2 D))^{2}} \# B(n-|w|) .
$$

Lemma 5. Let $r \geq 0$. There exists a number $F=F(G, A, r)>0$ such that for any finite set $X$ of elements of $G$ there is a subset $U \subseteq X$ such that $\# U \geq \frac{1}{F} \# X$ and $\left|x^{-1} y\right|>r$ for any distinct $x, y \in U$.

Proof. We take $F=\# B(r)$. Denote $B_{x}(r)=\left\{y \in G|| x^{-1} y \mid \leq r\right\}$. Clearly, $\# B_{x}(r)=\# B(r)$ for any $x \in G$. We choose subsequently arbitrary elements $x_{1}, x_{2}, \cdots \in G$ such that

$$
\begin{aligned}
x_{1} & \in X_{0}=X, \\
x_{2} & \in X_{1}=X \backslash B_{x_{1}}(r), \\
\ldots & \\
x_{k+1} & \in X_{k}=X \backslash \cup_{i=1}^{k} B_{x_{i}}(r),
\end{aligned}
$$

We have $\# X_{k} \geq \# X-k F$, so there are at least $\frac{1}{F} \# X$ such $x_{i}$ 's. We set $U=\left\{x_{1}, x_{2}, \ldots\right\}$.

\section{Proof of Theorems 2 and 3}

By Lemma 2(a), (c), for any $x, y \in G$, if $x$ is $C$-contained in $y$ then $x$ is strongly $(C+2 \delta)$-contained in $y$. As an immediate consequence, we obtain that Theorem 2 implies Theorem 3 . The rest of the section is devoted to proving Theorem 2 .

By a result of Coornaert [3], there exists $\alpha=\alpha(G, A)$ such that $\# B(n) \leq \alpha \lambda^{n}$ for all $n$. We introduce the following constants where 
$D$ and $E$ are as in Lemmas 3 and 4, respectively:

$$
\begin{gathered}
N_{0}=\log _{\lambda} 2 \alpha+2 D+E, \\
C_{1}=N_{0}+2 D+3 E+4 \delta, \\
C=C_{1}+9 D+5 E+8 \delta, \\
R=N_{0}+4 D+4 E+4 \delta .
\end{gathered}
$$

We fix an arbitrary $w \in G$ and assume that $\lambda_{w, C}=\lambda$. Our aim is to deduce a contradiction from this assumption.

Observe that any subword of a word in $\mathcal{L} \backslash \mathcal{L}_{w, C}$ lies in $\mathcal{L} \backslash \mathcal{L}_{w, C}$ as well. Since $\mathcal{L} \backslash \mathcal{L}_{w, C}$ forms a set of unique representatives for the elements in $G \backslash$ $Z_{w, C}$, this easily implies that $\#\left(B(n) \backslash Z_{w, C}\right)$ is a submultiplicative function on $n$. Then by [8, VI.C, Proposition 56], $\lambda_{w, C}=\inf _{n \geq 1} \#\left(B(n) \backslash Z_{w, C}\right)^{1 / n}$ and we consequently have $\#\left(B(n) \backslash Z_{w, C}\right) \geq \lambda^{n}$ for all $n$. Thus, with the assumption above, we have lower and upper bounds for \# $\left(B(n) \backslash Z_{w, C}\right)$ and $\# B(n)$ :

$$
\lambda^{n} \leq \#\left(B(n) \backslash Z_{w, C}\right) \leq \# B(n) \leq \alpha \lambda^{n} .
$$

Let $N$ be any number such that

$$
N \geq|w|+2 N_{0}+3 D+4 E+4 \delta .
$$

We reach a contradiction with (2) by proving that

$$
\lim _{k \rightarrow \infty} \frac{\#\left(B(k N) \backslash Z_{w, C}\right)}{\# B(k N)}=0 .
$$

To do this, we introduce two series $Y_{k}$ and $Y_{k}^{*}(k \geq 1)$ of subsets of $G$ :

$$
\begin{gathered}
Y_{k}=\{x \in G \mid x \text { does not }(C,(i-1) N, i N) \text {-contain } w \text { for } 1 \leq i \leq k-1, \\
x(C,(k-1) N, t) \text {-contains } w \text { for some } t \leq k N\}, \\
Y_{k}^{*}=\left\{x \in G \mid x \text { does not }\left(C_{1},(i-1) N, i N\right) \text {-contain } w \text { for } 1 \leq i \leq k-1,\right. \\
\left.x\left(C_{1},(k-1) N, t\right) \text {-contains } w \text { for some } t \leq k N\right\} .
\end{gathered}
$$

It immediately follows from the definition that $Y_{i}$ are pairwise disjoint and so are $Y_{i}^{*}$. We set

$$
Z_{k}=\bigcup_{i=1}^{k} Y_{i} .
$$

Clearly, $Z_{k} \subseteq Z_{w, C}$ for any $k$.

Lemma 6 (main lemma). There is a number $\beta>0$ such that for any $k \geq 1$ and $0 \leq i \leq k-1$,

$$
\#\left(Y_{i+1}^{*} \cap B(k N)\right) \geq \beta \#\left(B(i N) \backslash Z_{i}\right) \# B((k-i) N) .
$$


Before proving the lemma, we show how it implies (3) and hence proves Theorem 2. Since $Y_{i}^{*}$ are pairwise disjoint we have

$$
\sum_{i=0}^{k-1} \#\left(Y_{i+1}^{*} \cap B(k N)\right) \leq \# B(k N) .
$$

With the inequality in Lemma 6 this gives

$$
\beta \sum_{i=0}^{k-1} \#\left(B(i N) \backslash Z_{i}\right) \# B((k-i) N) \leq \# B(k N) .
$$

By submultiplicativity of \#B(n),

$$
\frac{\# B(k N)}{\# B(i N)} \leq \# B((k-i) N)
$$

and hence, after dividing by $\# B(k N)$ we obtain

$$
\beta \sum_{i=0}^{k-1} \frac{\#\left(B(i N) \backslash Z_{i}\right)}{\# B(i N)} \leq 1 .
$$

But since this holds for any $k$ and $Z_{i} \subseteq Z_{w, C}$, it follows that the series $\sum_{i=1}^{\infty} \frac{\#\left(B(i N) \backslash Z_{w, C}\right)}{\# B(i N)}$ converges thus implying (3) as required.

Proof of Lemma 6 . Let $k \geq 1$ and $0 \leq i \leq k-1$. For $m<n$ we denote

$$
S(m, n)=B(n) \backslash B(m) .
$$

By the definition of $N_{0}$, the inclusion $Z_{i} \subseteq Z_{w, C}$ and (2),

$$
\begin{aligned}
& \#\left(S\left(i N+2 D+E, i N+N_{0}\right) \backslash Z_{i}\right) \\
& \quad=\#\left(B\left(i N+N_{0}\right) \backslash Z_{i}\right)-\#\left(B(i N+2 D+E) \backslash Z_{i}\right) \\
& \quad \geq \lambda^{i N+N_{0}}-\alpha \lambda^{i N+2 D+E} \geq \alpha \lambda^{i N+2 D+E} \geq \#\left(B(i N) \backslash Z_{i}\right) .
\end{aligned}
$$

By Lemma 5, there is a number $F=F(G, A, R)>0$ and a subset $U \subseteq S\left(i N+2 D+E, i N+N_{0}\right) \backslash Z_{i}$ such that $\left|x^{-1} y\right|>R$ for any distinct $x, y \in U$ and

$$
\# U \geq \frac{1}{F} \#\left(B(i N) \backslash Z_{i}\right) .
$$

For any $x \in U$ we define a set $V_{x} \subset G$. By Lemma 3, there is a word $\hat{x} \in \mathcal{L}$ such that

$$
\hat{x} \equiv x_{1} x_{2} \quad \text { where } \quad\left|x^{-1} x_{1}\right| \leq D,\left|w x_{2}^{-1}\right| \leq D .
$$


We define, as in Lemma 4,

$$
V_{x}=\left\{y \in G|| \hat{x}^{-1} y_{1} \mid \leq E \text { for some initial segment } y_{1} \text { of } \bar{y}\right\} .
$$

We have $i N+2 D+E \leq|x| \leq i N+N_{0},|w|+|x|-2 D \leq|\hat{x}| \leq$ $|w|+|x|+2 D$ and hence

$$
i N+|w|+E \leq|\hat{x}| \leq i N+|w|+N_{0}+2 D .
$$

Since $k N \geq i N+N \geq i N+|w|+N_{0}+2 D$, by Lemma 4

$$
\#\left(V_{x} \cap B(k N)\right) \geq \kappa \#(B(k N-|\hat{x}|)) .
$$

Using the upper bound for $|\hat{x}|$ and submultiplicativity of $\# B(n)$ we get

$$
\begin{aligned}
\#(B(k N-|\hat{x}|)) & \geq \#\left(B\left((k-i) N-|w|-N_{0}-2 D\right)\right) \\
& \geq \frac{\#(B((k-i) N))}{\#\left(B\left(|w|+N_{0}+2 D\right)\right)} .
\end{aligned}
$$

This gives a lower bound for $\#\left(V_{x} \cap B(k N)\right)$ :

$$
\#\left(V_{x} \cap B(k N)\right) \geq \frac{\kappa}{\#\left(B\left(|w|+N_{0}+2 D\right)\right)} \#(B((k-i) N)) .
$$

We prove the following two assertions about the sets $V_{x}$ :

(i) $V_{x} \subseteq Y_{i+1}^{*}$ for any $x \in U$;

(ii) $V_{x} \cap V_{x^{\prime}}=\emptyset$ for $x \neq x^{\prime}$.

Observe that (i), (ii) and the lower bounds for $\#\left(V_{x} \cap B(k N)\right)$ and (5) for $\# U$ imply the desired inequality (4) for $\beta=\frac{\kappa}{F \#\left(B\left(|w|+N_{0}+2 D\right)\right)}$ in an obvious way. To prove the lemma, it remains to prove (i) and (ii).

Proof of (i). Let $x \in U$ and $y \in V_{x}$. We check for $y$ the conditions in the definition of $Y_{i+1}^{*}$. First we show that $y\left(C_{1}, i N, t\right)$-contains $w$ for some $t \leq(i+1) N$. Since $x_{2}$ in (6) is $D$-close to $w$, by Definition $2 \hat{x}\left(D,\left|x_{1}\right|,|\hat{x}|\right)$ contains $w$. Let $y_{1}$ be the initial segment of $\bar{y}$ with $\left|\hat{x}^{-1} y_{1}\right| \leq E$. By Lemma $2(\mathrm{f}), w$ is $\left(D+E+2 \delta, t_{1}, t_{2}\right)$-contained in $y_{1}$, and therefore in $y$, where $\left|t_{1}-\right| x_{1}|| \leq 2 E+2 \delta$ and $\left|t_{2}-\right| \hat{x}|| \leq 2 E+2 \delta$. Now

$$
\left|t_{1}-i N\right| \leq|| x_{1}|-i N|+2 E+2 \delta \leq|| x|-i N|+D+2 E+2 \delta \leq N_{0}+D+2 E+2 \delta
$$

and by the choice of $N$,

$$
\begin{aligned}
(i+1) N-t_{2} & \geq(i+1) N-2 E-2 \delta-|\hat{x}| \\
& \geq(i+1) N-2 E-2 \delta-i N-|w|-N_{0}-2 D \\
& \geq N_{0}+D+2 E+2 \delta .
\end{aligned}
$$


Observing that $|y| \geq|\hat{x}|-E \geq i N$ by the definition of $V_{x}$ and (7), we conclude by Lemma $2(\mathrm{e})$ that $w$ is $\left(N_{0}+2 D+3 E+4 \delta, i N, t\right)$-contained in $y$ for some $t \leq(i+1) N$ as required.

Now we prove that $y$ does not $\left(C_{1},(j-1) N, j N\right)$-contain $w$ for all $1 \leq j \leq i$. Assume that $y$ does $\left(C_{1},(j-1) N, j N\right)$-contain $w$ for some $1 \leq j \leq i$. Since

$$
j N-\left|y_{1}\right| \leq j N-|\hat{x}|+E \leq(j-i) N-|w|+2 D+E \leq 2 D+E,
$$

by Lemma 2(e) $w$ is $\left(C_{1}+2 D+E,(j-1) N, t\right)$-contained in $y_{1}$ for some $t$ with $j N-2 D-E \leq t \leq j N$. By Lemma 2(f), $w$ is $\left(C_{1}+2 D+\right.$ $\left.2 E+2 \delta, t_{1}, t_{2}\right)$-contained in $\hat{x}$ where $\left|t_{1}-(j-1) N\right| \leq 2 E+2 \delta$ and $\left|t_{2}-t\right| \leq 2 E+2 \delta$. We have

$$
\left|t_{2}-j N\right| \leq\left|t_{2}-t\right|+2 D+E \leq 2 D+3 E+2 \delta .
$$

Now $j N-\left|x_{1}\right| \leq j N-|x|+D \leq D$ and using Lemma 2(e) again we find that $w$ is $\left(C_{1}+3 D+2 E+2 \delta, t_{1}, t_{2}^{\prime}\right)$-contained in $x_{1}$ with $\mid t_{2}^{\prime}-$ $j N \mid \leq 3 D+3 E+2 \delta$. Another application of Lemma 2(f) gives that $w$ is $\left(C_{1}+4 D+2 E+4 \delta, t_{1}^{\prime}, t_{2}^{\prime \prime}\right)$-contained in $x$ where $\left|t_{1}^{\prime}-(j-1) N\right| \leq$ $2 D+2 E+4 \delta$ and $\left|t_{2}^{\prime \prime}-j N\right| \leq 5 D+3 E+4 \delta$. Again by Lemma $2(\mathrm{e}), w$ is $\left(C_{1}+9 D+5 E+8 \delta,(j-1) N, s\right)$-contained in $x$ for some $s \leq j N$. Due to the choice of $C$ and the definition of $Y_{k}$ this means that $x \in Y_{l}$ for some $l \leq j$. But this is a contradiction with $x \notin Z_{i}$. This finishes the proof of (i).

Proof of (ii). Let $y \in V_{x}$. By Lemma 2(b), $\left|x_{1}^{-1} y^{\prime}\right| \leq E+\delta$ for some initial segment $y^{\prime}$ of $\bar{y}$ and hence we have

$$
\left|x^{-1} y^{\prime}\right| \leq\left|x^{-1} x_{1}\right|+\left|x_{1}^{-1} y^{\prime}\right| \leq E+D+\delta .
$$

Assume that $y \in V_{x^{\prime}}$ for some $x^{\prime} \neq x$. Then there is another initial segment $y^{\prime \prime}$ of $\bar{y}$ such that $\left|\left(x^{\prime}\right)^{-1} y^{\prime \prime}\right| \leq E+D+\delta$. Since $x, x^{\prime} \in S\left(i N, i N+N_{0}\right)$ we have $\left\|x|-| x^{\prime}\right\| \leq N_{0}$ and therefore $\| y^{\prime}|-| y^{\prime \prime}|| \leq N_{0}+2 E+2 D+2 \delta$. Since both $y^{\prime}$ and $y^{\prime \prime}$ are initial segments of $\bar{y}$ this implies $\left|\left(y^{\prime}\right)^{-1} y^{\prime \prime}\right| \leq$ $N_{0}+2 E+2 D+2 \delta$ and finally we obtain $\left|x^{-1} x^{\prime}\right| \leq N_{0}+4 D+4 E+4 \delta$. But this contradicts the choice of $R$ and the definition of $U$. Lemma 6 is proved.

\section{References}

1. G. N. Arzhantseva, On quasiconvex subgroups of word hyperbolic groups, Geometriae Dedicata 85 (2001), 191-208.

2. M. R. Bridson, A. Haefliger, Metric spaces of non-positive curvature, Grundlehren der Mathematischen Wissenschaften [Fundamental Principles of Mathematical Sciences], 319, Springer-Verlag, Berlin, 1999. 
3. M. Coornaert, Mesures de Patterson-Sullivan sur le bord d'un espace hyperbolique au sens de Gromov, Pacific J. Math. 159 (1993), 241-270.

4. E. Ghys, P. de la Harpe (editors), Sur les groupes hyperboliques d'après Mikhael Gromov, Progress in Math. 83, Birkhäuser, 1990.

5. R. Grigorchuk, P. de la Harpe, On problems related to growth, entropy, and spectrum in group theory, J. Dynam. Control Systems 3 (1997) 1, 51-89.

6. M. Gromov, Metric Structures for Riemannian and Non-Riemannian Spaces, edited by J. Lafontaine, P. Pansu, Progress in Math. 152, Birkhäuser, 1999.

7. M. Gromov, Hyperbolic groups, in: Essays in group theory, ed. S.M. Gersten, M.S.R.I. Publ. 8, Springer, 1987, 75-263.

8. P. de la Harpe, Topics in geometric group theory, Chicago Lectures in Mathematics. University of Chicago Press, Chicago, IL, 2000.

9. A. Sambusetti, Growth tightness of free and amalgamated products, preprint. Universitá degli Studi di Roma "La Sapienza", 2001.

10. Z. Sela, Endomorphisms of hyperbolic groups. I. The Hopf property, Topology 38 (1999) 2, 301-321. 